\title{
Wide-bandwidth mode-hop-free tuning of extended-cavity GaN diode lasers
}

\author{
Johan Hult, lain S. Burns, and Clemens F. Kaminski
}

\begin{abstract}
We present a new approach for extended-cavity diode-laser tuning to achieve wide mode-hop-free tuning ranges. By using a multiple piezoactuated grating mount, the cavity length and grating angle in the laser can be adjusted independently, allowing mode-hop-free tuning without the need for a mechanically optimized pivot-point mount. Furthermore, synchronized diode injection-current tuning allows diode lasers without antireflection coatings to be employed. In combination these two techniques make the construction of a cheap, efficient, and easily optimized extended-cavity diode laser possible. A theoretical analysis is presented for optimal control of piezoactuator displacements and injection current to achieve the widest possible mode-hop-free tuning ranges, and a comparison is made with measurements. The scheme is demonstrated for blue and violet GaN lasers operating at $\sim 450 \mathrm{~nm}$ and $\sim 410 \mathrm{~nm}$, for which continuous tuning ranges exceeding $90 \mathrm{GHz}$ have been achieved. Examples of applications of these lasers are also given. (C) 2005 Optical Society of America

OCIS codes: $140.3600,140.2020,120.4570$.
\end{abstract}

\section{Introduction}

Single-mode continuous-wave (cw) laser sources with wide wavelength tuning ranges are very useful for high-resolution spectroscopy. ${ }^{1}$ Applications range from laser cooling ${ }^{2}$ to trace gas analysis, ${ }^{3}$ industrial process monitoring, ${ }^{4}$ and combustion diagnostics. ${ }^{5}$ For such applications it is essential that tuning is continuous, and free from mode-hops.

Diode lasers are increasingly gaining importance in this context, as they are compact, cheap, and robust. They are characterized by a relatively wide gain curve, resulting in a large tuning capability, achieved by varying injection current and/or temperature of the diode. Diode lasers are available from the midinfrared spectral region down to the violet part of the visible spectrum. The recent availability of commercial GaN-based laser diodes in the 370-480 nm wavelength range ${ }^{6,7}$ has made it possible to gain access to transitions of many species that have previously been impossible to directly probe using diode lasers.

By careful use of optical-feedback techniques it is possible to achieve a tunable narrow-linewidth laser

Johan Hult (jfh36@cheng.cam.ac.uk), Iain S. Burns, and Clemens F. Kamiski are with the Department of Chemical Engineering, Cambridge University, Pembroke Street, Cambridge CB2 3RA, UK.

Received 6 October 2004; accepted 20 January 2005.

0003-6935/05/183675-11\$15.00/0

(C) 2005 Optical Society of America source based on Fabry-Perot (FP) lasers, which are available at a wide range of wavelengths. ${ }^{1} \mathrm{~A}$ simple implementation of this idea is the external- or extended-cavity diode laser (ECDL), in which the first-order diffracted beam from a grating mounted in the Littrow configuration is used for feedback. ${ }^{8,9} \mathrm{~A}$ standard FP diode laser in the Littrow configuration can be coarsely tuned over its gain bandwidth through rotation of the grating. It will generally emit at a single narrow-linewidth longitudinal mode (typically $100 \mathrm{kHz}$ to $10 \mathrm{MHz}$ wide) and exhibit a much better spectral stability than the FP laser running without feedback.

In an ECDL, emission will take place where a longitudinal mode of the extended cavity matches an FP diode-laser mode that occurs close to the peak of the grating wavelength feedback profile. ${ }^{10}$ The FP diode-laser mode structure is determined by the cavity formed between the two diode facets, and FP modes are thus much more widely spaced in frequency than corresponding modes of the extended cavity (which is formed between the grating and the diode front facet). For continuous wavelength tuning, several laser parameters must be tuned in synchronization. When rotating the grating, the feedback wavelength profile shifts, and to avoid mode-hops, the laser modes must be shifted at the same rate by adjusting the cavity lengths, thus preserving the overlap. Furthermore, the two resonant optical fields in the FP and extended cavities must 
be kept in phase to avoid destructive interference and thus intensity losses.

Normally the grating is mounted on a lever arm, which is rotated around a carefully chosen pivot point to preserve the overlap between the extended-cavity mode and the grating-feedback center wavelength during wavelength scanning. ${ }^{11,12}$ A single piezoelectric actuator, or another electromechanical device, is then used to rotate the grating around its pivot point. However, it is also possible to use a multiplepiezoactuator optical mount to achieve the same relation between grating rotation and translation during tuning. ${ }^{13,14}$ The advantage of this approach is that the requirement for accurate mechanical positioning of the pivot point is replaced by a simple balancing of the electrical signals driving the piezoactuators. Furthermore, it is no longer necessary to construct a precision pivot-point grating mount, as a normal kinematic mount can be used. As cavity length and grating angle can be independently changed, it is also possible to optimize the overlap between the extended-cavity mode structure and the grating-feedback center wavelength before the wavelength tuning is commenced. Such an optimization is more difficult to achieve with the conventional pivotarm design.

In many ECDL systems, diodes with an antireflection coating on the output facet are used, with the aim of suppressing the oscillating optical field within the diode FP cavity. ${ }^{15} \mathrm{As}$ a result it should no longer be necessary to match the diode FP cavity length to have a continuously coinciding resonance with the extended cavity. In practice, however, it is still necessary to match the cavity lengths to achieve a large tuning range with antireflection-coated diodes, ${ }^{16}$ as the residual reflectance is generally not low enough to totally suppress the FP mode structure of the diode. The disadvantage with this approach is that coating of the diode is a complex and expensive process, and there is limited "off the shelf" availability of antireflection-coated diodes. However, by modulating the diode injection current, and thus modulating the effective diode-cavity length, in synchronization with the extended-cavity tuning, it is possible to match the diode FP and extended-cavity modes. ${ }^{9,17}$ Using this method, standard off-the-shelf diodes can be used, resulting in low-cost ECDL systems.

Here we report on the successful development and application of a new method that combines the advantages of multiple-actuator reflection-grating tuning and synchronized injection-current modulation, to achieve mode-hop-free tuning of a Littrow configuration ECDL. Commercially available uncoated standard diodes, optomechanical mounts, and laser drive electronics were combined to create, a simple and cost-efficient ECDL system. Two different ECDL systems operating at $\sim 450 \mathrm{~nm}$ and $\sim 410 \mathrm{~nm}$ have been constructed based on this principle. A number of ECDL systems operating in the violet spectral region, $390-420 \mathrm{~nm}$, have previously been reported, achieving a $10-50 \mathrm{GHz}$ tuning range. ${ }^{16,18-20}$ The two systems described here, however, have achieved mode-hop-free tuning ranges

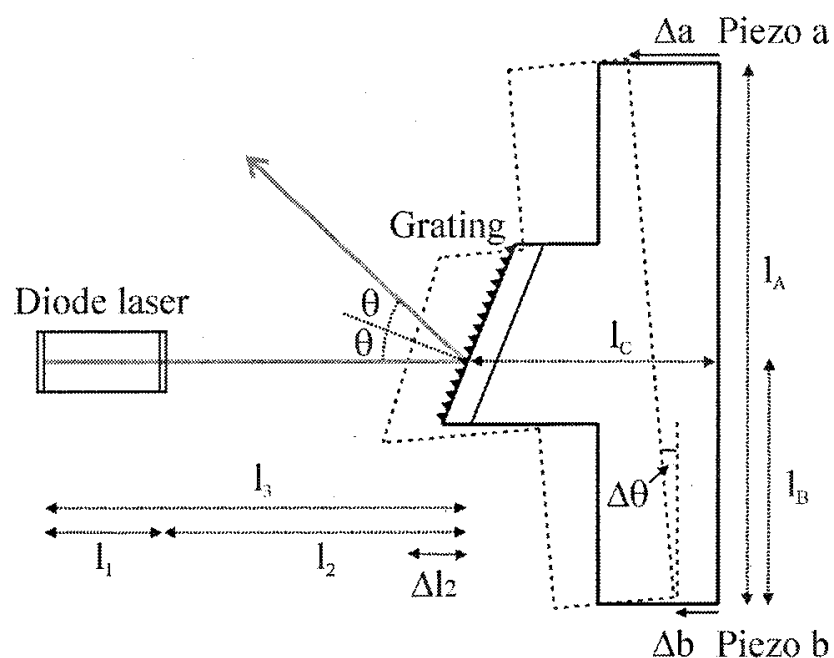

Fig. 1. Schematic illustration of the multiple-actuator extendedcavity diode-laser system.

of more than $110 \mathrm{GHz}$ and $90 \mathrm{GHz}$, respectively, which as far as we are aware, represent the widest tuning ranges reported for ECDL systems operating in the blue or violet spectral region.

In the first section of the paper a model is presented, which describes how electrical signals regulating piezoactuator displacements and diode injection current must be varied relative to one another, to achieve synchronized scanning of grating angle, extended-cavity length, and diode-cavity length for continuous wavelength tuning. This is followed by a detailed description of the multiple-piezo ECDL laser design. Results from a detailed characterization of the two ECDL systems constructed are then presented, and the results are compared to model predictions. Finally, an example of an application of the laser systems is presented, followed by conclusions.

\section{Experimental Design}

\section{A. Mode-Hop-Free Tuning}

In a standard Littrow ECDL arrangement a reflection grating is used to feed the first-order $(m=-1)$ beam back into the diode laser, whereas the zerothorder forms the output beam of the device (see Fig. 1). The grating angle, $\theta$, determines the feedback wavelength, and thus also the output wavelength of the diode laser. If the feedback is sufficiently strong and overlaps with an FP mode, a single mode will be emitted. For the Littrow configuration the following condition must be satisfied:

$$
2 d \sin \theta=-\lambda,
$$

where $d$ is the grating period, $\lambda$ is the laser wavelength, and $\theta$ is the angle of incidence onto the grating. Wavelength tuning is performed by rotation of the grating. However, to maintain single-mode operation and to avoid mode-hops, the length $l_{2}$ of the extended cavity (formed by the grating and the diode 
front facet) must be synchronously adjusted such that the number of longitudinal modes $N_{2}$ sustained in the cavity remains constant:

$$
l_{2}=N_{2} \lambda / 2 n,
$$

where $n$ is the index of refraction of the medium inside the cavity, which is normally air and thus assumed to be $n=1$. Two piezocrystal actuators are indicated as $a$ and $b$ in Fig. 1, and they allow adjustments of both cavity length, $l_{2}$, and grating angle, $\theta$. Firstly, the relative motion of $a$ and $b$ is considered such that mode-hop-free tuning of the extended cavity is ensured. It should be noted that in the present realization of the ECDL there are in fact three piezoactuators controlling the grating. However, the second and third actuators, $b$ and $c$, are positioned at different locations along the same vertical axis (see Fig. 3 for a three-dimensional view of the arrangement), and they are driven with the same control signal. Thus $b$ and $c$ always move in synchronicity and are therefore considered as a single actuator, denoted as $b$ in Fig. 1 . The change in grating angle $\Delta \theta$ resulting from piezoactuator expansions of $\Delta a$ and $\Delta b$ is given by

$$
\tan (\Delta \theta)=\frac{\Delta a-\Delta b}{l_{A}}
$$

where $l_{A}$ is the distance between the two actuator tips. Differentiation of Eq. (1) gives

$$
\Delta \theta=-\frac{\Delta \lambda}{2 d \cos (\theta)}
$$

Substituting Eq. (4) into Eq. (3) gives

$$
\Delta a-\Delta b=l_{A} \tan \left(\frac{-\Delta \lambda}{2 d \cos (\theta)}\right) \approx \frac{-l_{A}}{2 d \cos (\theta)} \Delta \lambda
$$

where the last simplification is valid for small values of $\Delta \theta$.

Figure 2 shows the movement of the grating, with respect to the incident laser beam, for a change in angle $\Delta \theta$. The grating displacement along the laser beam axis is $\Delta l_{2 \text {, real }}$; however, there is an accompanying translation, $\Delta t$, across the laser beam axis in the grating plane. A grating translation in the plane of the grating introduces a phase change $\Delta \phi=$ $2 \pi \Delta t / d$ of the diffracted beam, where $d$ is the grating period. ${ }^{12}$ Thus there will be $\Delta t / d$ mode-hops without a compensation mechanism. The grating translation $\Delta t$ can be split into two components, one perpendicular to the laser beam and one parallel to the beam. The perpendicular translation component will not affect the wavelength tuning of the laser. ${ }^{21}$ The parallel translation component causes an increase of the extended-cavity length. To account for the grating translation effect, the change in effective extendedcavity length $\Delta l_{2 \text {, eff }}$ must be considered (equivalent to

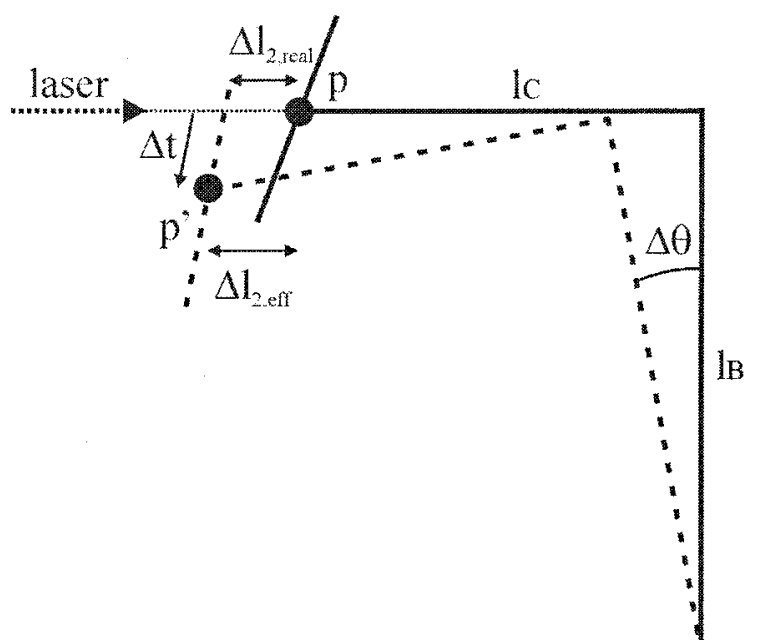

Fig. 2. Detailed view of the grating translation with respect to the incident laser beam, as the angle of the grating is changed.

$\Delta l_{2}$ in Fig. 1), which is given by

$$
\begin{aligned}
\Delta l_{2} & =\Delta l_{2, \text { eff }}=l_{C}(1-\cos (\Delta \theta))-l_{B} \sin (\Delta \theta)-\Delta b \\
& \approx-l_{B} \Delta \theta-\Delta b=\frac{l_{B}}{2 d \cos (\theta)} \Delta \lambda-\Delta b
\end{aligned}
$$

where the first two terms come from the change in angle, $\Delta \theta$, and correspond to the horizontal movement of point $p$ to $p^{\prime}$ in Fig $2 . l_{B}$ is the distance between the tip of actuator $b$ and the laser beam axis, in the direction perpendicular to the laser beam, and $l_{C}$ is the distance between the plane defined by the actuator-tip contact points and the point where the laser strikes the grating.

Differentiation of Eq. (2) gives

$$
\Delta l_{2}=\frac{N_{2}}{2} \Delta \lambda=\frac{l_{2}}{\lambda} \Delta \lambda
$$

which corresponds to the change in extended-cavity length that is needed to compensate for a particular change in wavelength. Substitution into Eq. (6) gives

$$
\Delta b=\frac{l_{B}}{2 d \cos (\theta)} \Delta \lambda-\frac{l_{2}}{\lambda} \Delta \lambda
$$

which, used with Eq. (5), results in the following expression for the expansion $\Delta a$ required of the other piezo:

$$
\Delta a=\frac{-l_{A}}{2 d \cos (\theta)} \Delta \lambda+\frac{l_{B}}{2 d \cos (\theta)} \Delta \lambda-\frac{l_{2}}{\lambda} \Delta \lambda
$$

Dividing Eq. (9) by Eq. (8) gives the ratio between the changes to these two piezoactuators needed to preserve mode-hop-free tuning of the extended cavity: 


$$
\begin{aligned}
\frac{\Delta a}{\Delta b} & =\frac{l_{B}-l_{A}-2 l_{2} d \cos (\theta) / \lambda}{l_{B}-2 l_{2} d \cos (\theta) / \lambda}=1+\frac{l_{A}}{2 l_{2} d \cos (\theta) / \lambda-l_{B}} \\
& =1+\frac{l_{A}}{2 l_{2} d \sqrt{1-\sin ^{2}(\theta) / \lambda-l_{B}}} \\
& =1+\frac{l_{A}}{l_{2} \sqrt{\frac{4 d^{2}}{\lambda^{2}}-\left(\frac{2 d \sin (\theta)}{\lambda}\right)^{2}}-l_{B}} \\
& =1+\frac{l_{A}}{l_{2} \sqrt{\frac{4 d^{2}}{\lambda^{2}}-1}-l_{B}} .
\end{aligned}
$$

Neglecting effects of nonlinearity and hysteresis, the expansion of each piezoactuator $\Delta l_{x}$ is assumed to be proportional to the voltage applied to the piezocrystal $V_{x}\left(\Delta l_{x}=\alpha V_{x}\right)$. If the two actuators have the same value of $\alpha$, the ratio between the voltages applied to the piezos $\Delta V_{a} / \Delta V_{b}$ is identical to Eq. (10). This means that to achieve a linear wavelength scan with the ECDL, two linear voltage ramps should be applied to piezoactuator $a$ and to piezoactuators $b$ and $c$ respectively, with the ratio between the slopes of the two ramps given by Eq. (10).

Furthermore, the length of the diode chip cavity $l_{1}$, formed by the front and back facets, must also be tuned in synchronization with the grating, to preserve the number of longitudinal modes $N_{1}$ within the diode cavity:

$$
l_{1}=N_{1} \lambda / 2 n
$$

where $n$ is the index of refraction of the diode crystal. Failure to match the diode cavity to the extended cavity will lead to destructive interference between the light in the two cavities, and failure to match the diode cavity to the grating feedback wavelength will lead to mode-hops during wavelength tuning of the ECDL. There is also a third cavity present, formed by the back facet of the diode and the grating $\left(l_{3}=l_{1}\right.$ $+l_{2}$; see Fig. 1). However, if the number of longitudinal modes in the diode and extended cavities, $l_{1}$ and $l_{2}$, are preserved during scanning, the number of modes in cavity $l_{3}$ is automatically also preserved.

The length of the diode cavity $l_{1}$ is adjusted by tuning the diode laser current $I$, which has the effect of increasing the diode-junction temperature through Joule heating. If there is a simple relationship between the required diode current and piezoactuator modulation signals, then the ECDL scan control signals could all be derived from a common signal source. Assuming a linear dependence of effective diode-chip length on injection current, one can define a parameter $\beta$ expressing the change in wavelength with the diode-laser injection current:

$$
\Delta \lambda=\beta \Delta I=\frac{2 n}{N_{1}} \Delta l_{1}
$$

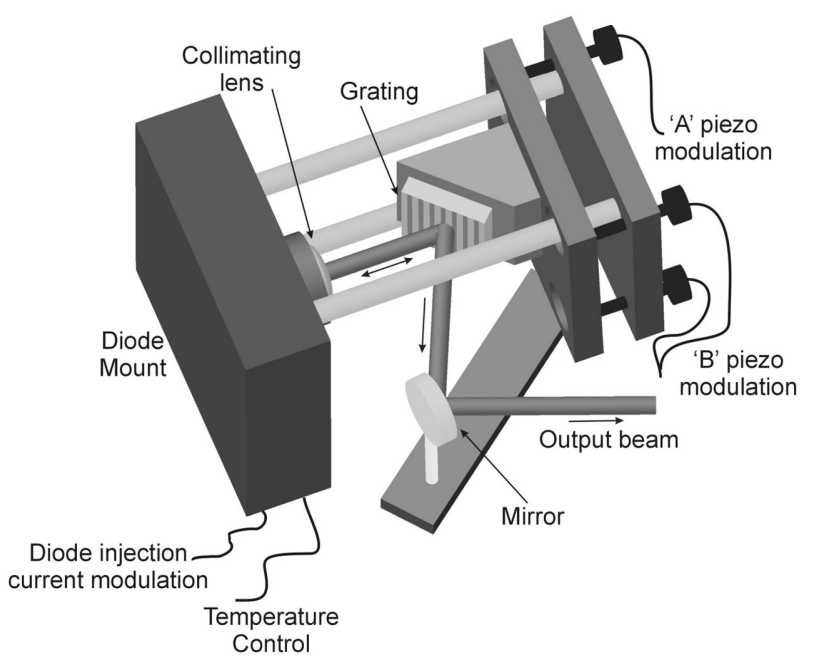

Fig. 3. Schematic illustration of the extended-cavity diode-laser system.

where the last step is taken by differentiation of Eq. (11). This relationship combined with Eq. (8) gives the ratio between the current modulation, $\Delta I$, and piezomodulation, $\Delta V_{b}$ :

$$
\frac{\Delta I}{\Delta V_{b}}=\frac{\frac{1}{\beta} \Delta \lambda}{\frac{1}{\alpha} \Delta b}=\frac{\alpha}{\beta\left(\frac{l_{B}}{2 d \cos \theta}-\frac{l_{2}}{\lambda}\right)}=\frac{\alpha \lambda}{\beta\left(\frac{l_{B}}{\sqrt{\frac{4 d^{2}}{\lambda^{2}}-1}-l_{2}}\right)} .
$$

Therefore, it is possible to achieve a linear mode-hopfree wavelength scan with the ECDL, by scanning the laser-diode current using a linear ramp synchronized to the piezoactuator driving signals. The ratio of the slope of the current ramp and the voltage ramp driving piezoactuator $b$ is given by Eq. (13). As the last term of Eq. (13) is negative for a typical ECDL setup, the diode-current slope should have opposite sign to the piezovoltage slopes.

\section{B. Experimental Setup}

The diode laser used in the first ECDL system was a commercial multimode FP GaN device (Nichia Corporation, NDHB500APAE1) operating at $\sim 450 \mathrm{~nm}$, with a maximum power rating of $5 \mathrm{~mW}$. The laser used in the second ECDL was also a FP GaN device (Nichia Corporation, NDHV310APB). At room temperature it operates at $\sim 410 \mathrm{~nm}$, with a maximum power of $30 \mathrm{~mW}$. The original front facet reflectivities of these commercial devices were not modified; for devices similar to the $410 \mathrm{~nm}$ laser it has been estimated to be $\sim 4 \% .^{22}$

The extended cavity was constructed from commercially available components; see Fig 3 . The diode laser was mounted in a thermoelectrically controlled laser-diode mount (Thorlabs, TCLDM9). 

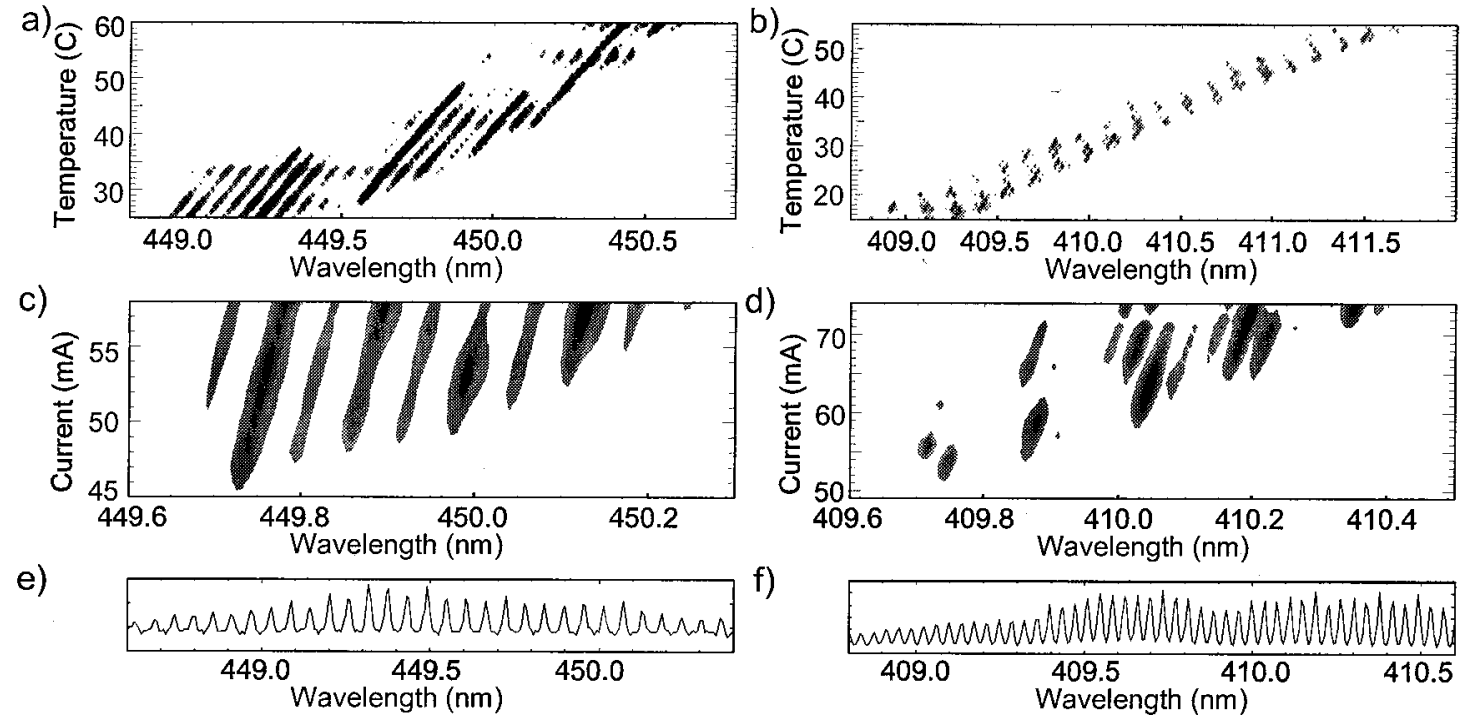

Fig. 4. Spectral output of the Fabry-Perot diode lasers as a function of (a) temperature for the 450 nm laser, (b) temperature for the $410 \mathrm{~nm}$ laser, (c) current for the $450 \mathrm{~nm}$ laser, and (d) current for the $410 \mathrm{~nm}$ laser. Spectral output below lasing threshold for (e) the $450 \mathrm{~nm}$ diode and (f) the $410 \mathrm{~nm}$ diode.

An antireflection-coated molded glass aspheric lens (Thorlabs, C230TM-A, $f=4.5 \mathrm{~mm}, \mathrm{NA}=0.55$ ) was used to collimate the output beam from the laser. A holographic 1800 lines/mm grating (Edmund Scientific 43775) was mounted at $24.0^{\circ}$ for the $450 \mathrm{~nm}$ laser, and at $21.7^{\circ}$ angle for the $410 \mathrm{~nm}$ laser, in a piezoelectric kinematic mount (Thorlabs, KCl-PZ), which was attached directly to the laser-diode mount using three steel rods. The mount incorporated three piezoelectric actuators, permitting full rotational and translational movement of the grating. In the Littrow configuration the backreflection of the first-order beam was retroreflected into the cavity, and the zeroth-order served as the output. The reflection efficiency in first order was $\sim 75 \%$ for incident polarization oriented in the $s$ plane, which is the expected polarization of the diode output, and $\sim 45 \%$ for $p$ plane polarization. The output beam was reflected by a mirror mounted on the same plate as the grating, as shown in Fig. 3, thus ensuring that the direction of the beam remained constant even for large changes in grating angle associated with coarse wavelength tuning. ${ }^{23}$

The laser current and temperature were controlled by low-noise laser-diode drivers (Tektronix, LDC8002 and TED 8020). A three-channel piezoelectric driver (Thorlabs, MDT693) was used to control the displacement of the piezoelectric actuators of the kinematic mount. A signal generator was used to generate the triangular wave that controlled the ECDL frequency scan. The signal generator output was then split into three identical triangular waves with independently adjustable amplitudes, which were used to modulate the voltages on the piezoactuators and the laser-diode current. This splitting was achieved using a passive voltage dividing circuit incorporating one variable resistor for each of the three triangular waves.

The relative frequency change of the ECDL output during scans was measured by monitoring the transmission through a solid quartz Fabry-Perot etalon with a low finesse and a free spectral range (FSR) of $3.1 \mathrm{GHz}$, using a photodiode. In addition, a scanning $\mathrm{FP}$ etalon $(\mathrm{FSR}=7.5 \mathrm{GHz}, \mathrm{F}=30$ ) was used to monitor the spectral output of the ECDL to ensure that single-mode operation was maintained during scans. The power of the ECDL output beam was also measured, by directing a reflection from a quartz plate onto a second photodiode. Further characterization of the spectral output of the FP diode lasers was performed using a $1.26 \mathrm{~m}$ spectrometer with a 2400 lines/mm grating operating in first order, fitted with a backilluminated unintensified CCD camera for signal detection.

\section{Results}

Figure 4 shows the spectral output of the FabryPerot diode lasers, before mounting in the ECDL configuration, as a function of temperature and injection current, as well as the spectral output below laser threshold. From such temperature and current tuning maps, the parameters needed for the evaluation of the piezoactuator and current tuning rates were extracted.

Figure 4(a) shows a contour plot of the spectral output of the $450 \mathrm{~nm}$ FP diode laser for diode temperatures ranging from 25 to $60{ }^{\circ} \mathrm{C}$, when the laser is operating at a fixed injection current. From this plot it is apparent that, for most temperatures, 5-15 FP diode modes are active, whereas single-mode emission is only achieved within a very narrow range of operating conditions. The individual modes appear to be active and tune continuously with temperature in $10-20{ }^{\circ} \mathrm{C}$ long intervals. From the slope of the individual modes a wavelength tuning rate of $16 \mathrm{pm} /{ }^{\circ} \mathrm{C}$ can be estimated. The shift of the gain curve to higher wavelengths with increasing temperatures is also ap- 
parent $\left(\sim 40 \mathrm{pm} /{ }^{\circ} \mathrm{C}\right)$ as the overall mode pattern is shifting to the right. In Fig. 4(b) the corresponding plot for the $410 \mathrm{~nm}$ FP laser is shown. There are several distinct differences compared to the behavior of the $450 \mathrm{~nm}$ diode. For most temperatures there are only a few modes active, often separated by a multiple of the expected mode spacing. For a certain temperature there are typically 2-4 groups of active modes, each consisting of one or two neighboring modes. These groups are separated by gaps spanning two or three inactive modes. The cause of this spacing between groups of modes active at a specific temperature becomes apparent when studying the temperature tuning of the individual modes. Individual modes tune to higher wavelengths with increasing temperature at a rate of $\sim 15 \mathrm{pm} /{ }^{\circ} \mathrm{C}$. Most modes tune for $\sim 3{ }^{\circ} \mathrm{C}$ after first appearing; they then disappear and become inactive for $\sim 3{ }^{\circ} \mathrm{C}$ before reappearing again and tuning for another $3^{\circ} \mathrm{C}$. The gaps in the center of each mode tuning thus lead to the spacing between the groups of modes observed in Fig. 4(b). From the overall slope of the mode pattern seen in this figure, the rate at which the gain curve changes with temperature was estimated to be $\sim 60 \mathrm{pm} /{ }^{\circ} \mathrm{C}$, which is thus higher than the tuning rate of the individual modes, just as it was for the $450 \mathrm{~nm}$ laser.

The effect of current tuning on the spectral output of the FP diode lasers is shown in Figs. 4(c) and 4(d). With increasing injection current a larger number of modes become active, and the positions of the individual modes also shift to higher wavelengths $(\beta=$ $4.3 \mathrm{pm} / \mathrm{mA}$ for the $450 \mathrm{~nm}$ laser, and $\beta=4.0 \mathrm{pm} /$ $\mathrm{mA}$ for the $410 \mathrm{~nm}$ laser).

Figure 4(e) shows the spectral output of the $450 \mathrm{~nm}$ diode just below lasing threshold. The FP diodecavity mode structure imposed on the output emission is clearly seen. The mode spacing corresponds to a FSR of $58 \mathrm{pm}$, which would infer a chip length of $\sim 0.58 \mathrm{~mm}$, based on reported values for the index of refraction and dispersion of $\mathrm{GaN}$ at $450 \mathrm{~nm} .{ }^{24}$ The corresponding spectrum for the $410 \mathrm{~nm}$ laser is shown in Fig. 4(f). Here the FSR is $38 \mathrm{pm}$, corresponding to a chip length of $\sim 0.64 \mathrm{~mm}$, based on the properties of GaN at $410 \mathrm{~nm} .{ }^{24}$ These estimates of cavity length match those previously reported, ${ }^{7}$ and we found no evidence of any anomalous mode spacing in these devices, as has been observed, for example, by Jiang et al. ${ }^{25}$

When operated in the extended-cavity configuration, a reduction in the injection-current threshold for lasing from $40 \mathrm{~mA}$ to $37 \mathrm{~mA}$ is observed for the $450 \mathrm{~nm}$ diode. The laser-diode injection current and the extended-cavity piezoactuator voltages were then varied in synchronicity to achieve mode-hop-free wavelength tuning. Figure 5(a) shows an FP interferometer fringe pattern corresponding to a $110 \mathrm{GHz}$ wide scan of the $450 \mathrm{~nm}$ ECDL. The scan was accomplished by simultaneously modulating piezoactuators $a$ and $b$, and the diode-injection current $I$, as discussed in Section 2 . The scan was recorded in $250 \mathrm{~ms}$, with the laser operating at $25^{\circ} \mathrm{C}$. Two different FP interferometers $(\mathrm{FSR}=3.1 \mathrm{GHz}$ and $7.5 \mathrm{GHz}$ ) were used to monitor the laser frequency during the scan. The FP etalon transmission traces have been normalized by the simultaneously recorded laser output power, which is also shown.

During the scan shown in Fig. 5(a) the $7.5 \mathrm{GHz}$ etalon was used with a fixed mirror separation. In a second experiment, performed to validate singlemode emission during the entire ECDL scan, the etalon mirror spacing was scanned 25 times during each laser wavelength scan [see Fig. 5(b)]. Four of the resulting FP etalon scans are shown in Figs. 5(c)-5(f). The length of these FP etalon scans was adjusted to $\sim 2 \times$ FSR of the etalon, to ensure that the same laser mode appeared at least twice during each scan. In each of Figs. 5(c)-5(f) two peaks separated by the FSR of the etalon are clearly seen, corresponding to a single mode being emitted by the ECDL at each of the positions of the laser wavelength scan. Single-mode ECDL emission over the entire $110 \mathrm{GHz}$ wavelength scan is confirmed by the absence of side-modes in all 25 FP etalon scans.

As a comparison, previous ECDL designs employing antireflection-coated GaN diodes, pivot-point grating tuning, and current tuning have achieved a continuous tuning range of $\sim 50 \mathrm{GHz} .{ }^{16}$ Uncoated diodes in combination with optimized pivot-point grating mounting and current modulation, on the other hand, have achieved continuous tuning ranges in the range of $10-25 \mathrm{GHz} .{ }^{19}$ Finally, uncoated diodes in combination with unoptimized pivot-point grating mounting have typically achieved continuous tuning ranges below $10 \mathrm{GHz} .18,20$

The output power of the laser is also shown in Fig. 5 (a); it decreases to almost zero during the $110 \mathrm{GHz}$ long scan shown here. Such a decreasing laser output power during scanning is an inherent feature of all ECDLs employing current tuning. A higher output power at the end of the scan can of course be achieved by increasing the mean injection current or by decreasing the length of the scan. In the present case the injection current was modulated around a mean value of $51.9 \mathrm{~mA}\left(I_{\max }=54 \mathrm{~mA}\right)$, resulting in an output power from the ECDL of $\sim 0.9 \mathrm{~mW}$ at the centre of the scan.

For the $450 \mathrm{~nm}$ ECDL the extended cavity and diode parameters (defined in Fig. 1) were $l_{a}=45.0 \mathrm{~mm}, l_{b}$ $=22.5 \mathrm{~mm}, l_{2}=24.0 \mathrm{~mm}, \alpha=41 \mathrm{~nm} / \mathrm{V}$, and $\beta=$ $4.25 \mathrm{pm} / \mathrm{mA}$. This leads to the following predicted ratios between the modulation voltages for optimized tuning [Eqs. (10) and 13]: $\Delta V_{a} / \Delta V_{b}=2.43$, and $\Delta I / \Delta V_{b}=-0.27 \mathrm{~mA} / \mathrm{V}$.

To achieve the $110 \mathrm{GHz}$ wide mode-hop-free scan shown in Fig. 5, both modulation ratios have to be accurately adjusted; for the present ECDL they were $\Delta V_{a} / \Delta V_{b}=1.56$ and $\Delta I / \Delta V_{b}=-0.27 \mathrm{~mA} / \mathrm{V}$. It was found, however, that a wide range of ratios between $\Delta V_{a}$ and $\Delta V_{b}$ still resulted in fairly long mode-hopfree tuning ranges, as ranges exceeding $80 \mathrm{GHz}$ were observed for $\Delta V_{a} / \Delta V_{b}$ ranging from 1 to 2.5. This indicates that precise tuning of the grating angle is of less importance to achieve intermediate scanning ranges in the present setup, where, instead, exact 

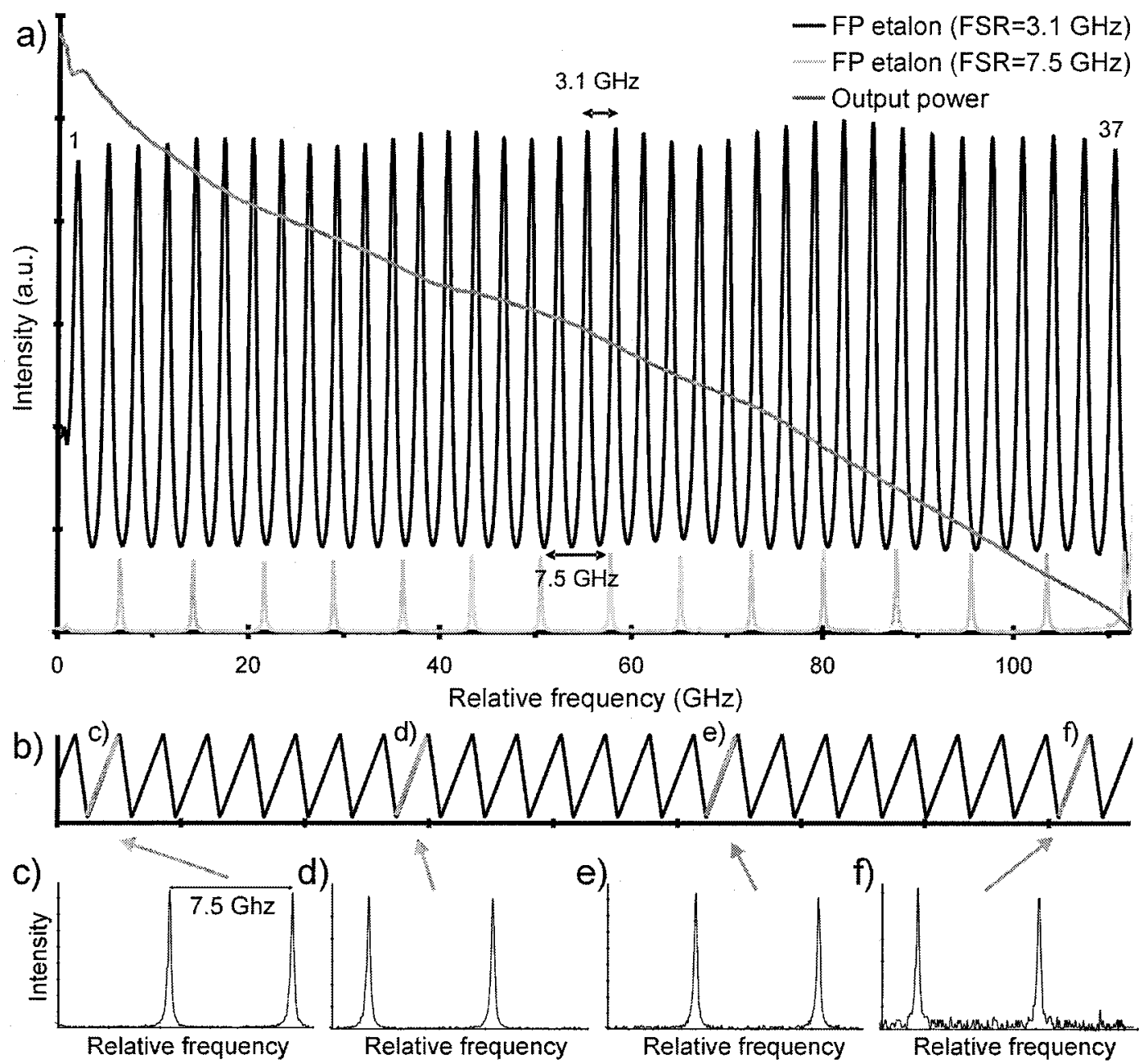

Fig. 5. (a) Transmitted fringe pattern of a $450 \mathrm{~nm}$ ECDL wavelength scan through two different Fabry-Perot interferometers (FSR $=3.1 \mathrm{GHz}$ and $7.5 \mathrm{GHz}$ ). 37 fringes from the lower FSR etalon are observed, corresponding to $110 \mathrm{GHz}$ mode-hop-free tuning. The corresponding laser output power is also shown. (b) Mirror spacing of scanning Fabry-Perot interferometer (FSR = 7.5 GHz) used to validate single-mode emission during ECDL scanning. (c)-(f) Scanning Fabry-Perot traces recorded at four different times during a single laser wavelength scan; the corresponding times are indicated in (b).

matching of the tuning of the extended-cavity length and diode-cavity length is more critical. The front facet reflectivity of the present diode is not negligible, and the grating wavelength feedback profile is relatively wide compared to the extended-cavity mode spacing. In Fig. 6 a schematic diagram of the modes and the grating-feedback profile for the present laser is shown. As a result the overlap between the active diode mode and the active extended-cavity mode required $^{10}$ is of larger importance than the exact over-

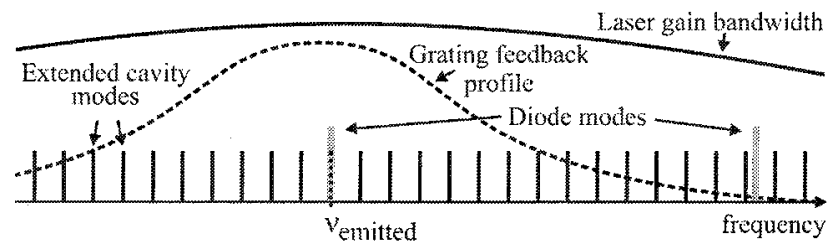

Fig. 6. Schematic diagram of the diode- and extended-cavity mode structure of an ECDL; the grating-feedback profile is also indicated. lap between the peak of the grating feedback and the active modes to preserve mode-hop-free single-mode emission. Further evidence for the minor importance of grating-angle tuning in the present ECDL is the fact that mode-hop-free tuning is achieved even when tuning the grating-feedback profile in the opposite direction to the extended-cavity mode tuning. A lower estimate of the full width at half-maximum of the grating profile is $67 \mathrm{GHz}$, obtained using the assumptions outlined by Boggs et al. ${ }^{26}$ It should be noted that the grating-feedback profile is still sufficiently narrow though, to favor only one of the diode FP modes. It is expected that the use of a higher-dispersion grating would increase the necessity to exactly match the grating-angle tuning to the cavity tuning. The experimentally determined extended-cavity mode spacing of the $450 \mathrm{~nm}$ ECDL was $6.2 \mathrm{GHz}$, which matches the spacing expected from a $24 \mathrm{~mm}$ long cavity.

It should be noted that great care must be taken to synchronize the grating tuning with the extended 

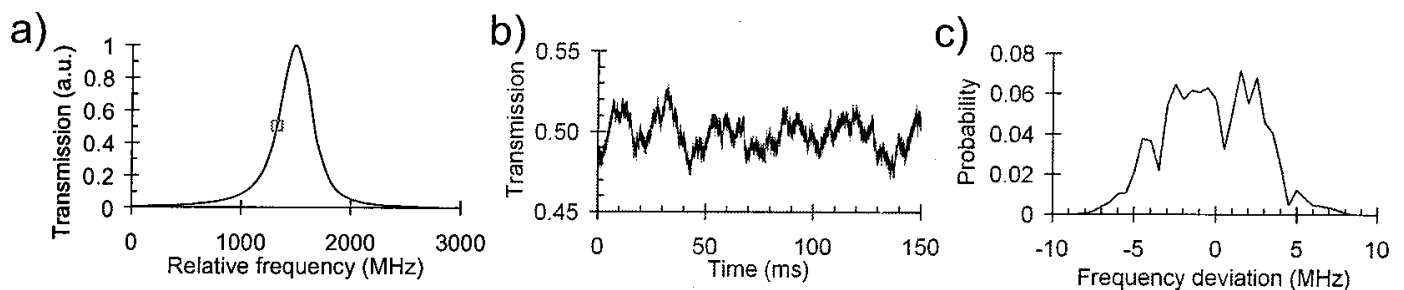

Fig. 7. (a) FP etalon transmission as a function of laser wavelength, (b) time series of FP etalon transmission, with the laser tuned to the location indicated by the gray box in (a), (c) histogram of instantaneous laser frequencies corresponding to the transmission trace in (b). From the width of the frequency distribution in (c) the $450 \mathrm{~nm}$ ECDL line width was determined to be below $8 \mathrm{MHz}$ over a $150 \mathrm{~ms}$ interval.

cavity length and current tuning when using either antireflection-coated diodes or gratings providing a narrower feedback profile. In contrast to the gratingangle tuning, the exact matching of the diode-current and extended-cavity length tuning is critical to achieve even intermediate mode-hop-free tuning ranges in the present ECDL. The theoretical ratio between current and piezotuning given by Eq. (13) is of course only valid when the piezos $a$ and $b$ are tuned by the ratio prescribed by Eq. (10). For tuning employing a different ratio between piezoactuator $a$ and $b$ elongations, such as for the scan in Fig. 5(a), for example, the tuning of the laser current should be matched to the effective cavity-length change [Eq. (6)], and in this more general case the optimum current tuning is given by

$$
\frac{\Delta I}{\Delta V_{b}}=\frac{\alpha \Delta \lambda}{\beta \Delta b}=\frac{\alpha \lambda \Delta l_{2}}{\beta l_{2} \Delta b}=\frac{\alpha \lambda}{\beta l_{2}}\left(\frac{l_{B}}{l_{A}}\left(1-\frac{\Delta V_{a}}{\Delta V_{b}}\right)-1\right),
$$

which is derived by combining Eqs. (3), (6), and (7). For the ratio $\Delta V_{a} / \Delta V_{b}=1.56$ used for the scan in Fig. 5(a), Eq. (14) predicts $\Delta I / \Delta V_{b}=-0.23$, which is close to the experimentally observed ratio of -0.27 . This close agreement was observed for a wide range of values of $\Delta V_{a} / \Delta V_{b}$.

Several factors limit the maximum tuning range of the present ECDL. When the ECDL is tuned using a ratio $\Delta V_{a} / \Delta V_{b} \approx 2.4$, it is limited to a value of $110 \mathrm{GHz}$ by the maximum displacement of piezoactuator $a(\sim 6 \mu \mathrm{m})$. On the other hand, when it is tuned using a ratio $\Delta V_{a} / \Delta V_{b} \approx 1$, it is limited by the FSR of the diode chip (to $\sim 85 \mathrm{GHz}$ ), as a further elongation of the diode cavity without an associated change in grating angle would lead to a better overlap between the grating-feedback profile and one of the neighboring diode-laser modes, thus forcing a mode-hop. For intermediate values of $\Delta V_{a} / \Delta V_{b}$, however, tuning ranges longer than $110 \mathrm{GHz}$ could be possible. However, in this intermediate region the limitation for the present $450 \mathrm{~nm}$ ECDL is the fact that the laser current is tuned below the threshold for lasing at the end of the scan, as is seen in Fig. 5. Lasers allowing wider current tuning ranges are thus expected to achieve longer scans, unless limitations are then instead imposed by nonlinearities in either piezoactuator response or current tuning, which prevent sufficient matching of extended cavity expansion and diodechip mode tuning over longer ranges.
The scan shown in Fig. 5 was recorded at a laser scan rate of $4 \mathrm{~Hz}$, to allow a sufficient number of $\mathrm{FP}$ etalon scans to be recorded during one wavelength sweep. However, the same tuning ranges were achieved at laser scanning rates up to $60 \mathrm{~Hz}$, above which the maximum tuning range gradually decreases with increasing scanning frequency. At $300 \mathrm{~Hz}$ the maximum mode-hop-free tuning range obtained was $15 \mathrm{GHz}$, whereas above $300 \mathrm{~Hz}$, useful tuning ranges were not observed. This decrease is due to mechanical resonances, piezoresponse nonlinearities, and limited diode heat transfer.

An upper estimate of the linewidth of the extendedcavity diode laser was determined by analyzing the fluctuations in transmission through a FP etalon (FSR $=7.5 \mathrm{GHz}, \mathrm{F}=30$ ) when the laser was tuned to the slope of one of its transmission peaks; see Fig. 7(a). ${ }^{14}$ At this position, fluctuations in laser frequency will appear as fluctuations in transmitted intensity; see Fig. 7(b). Using the slope of the transmission function at the indicated position in Fig. 7(a), the observed fluctuations in transmission could be converted to fluctuations in laser emission frequency. In Fig. 7(c) the histogram of instantaneous laser frequencies is shown. The width of this frequency histogram provides an upper estimate of the line width of $8 \mathrm{MHz}$, over a time period of $150 \mathrm{~ms}$. Note that in this approach, fluctuations in both FP etalon cavity length and laser power $(\sim 0.1 \%)$ also contribute to the measured line width, and the value reported here forms an estimate of the maximum possible line width.

A similar analysis was performed for the $410 \mathrm{~nm}$ device. In Fig. 8 a $93 \mathrm{GHz}$ long scan of the singlemode $410 \mathrm{~nm}$ ECDL is shown. During this scan, which was achieved in $50 \mathrm{~ms}$, the ECDL output energy drops by $\sim 45 \%$, as a result of the diode injectioncurrent tuning. The current was modulated around a mean value of $70 \mathrm{~mA}\left(I_{\max }=80 \mathrm{~mA}\right)$, resulting in an output power from the ECDL of $\sim 2 \mathrm{~mW}$. Just as for the $450 \mathrm{~nm}$ ECDL described above, relatively large mode-hop-free tuning ranges can be achieved with this laser for a wide range of ratios between $\Delta V_{a}$ and $\Delta V_{b}$. For the scan shown in Fig. 8 the actual ratios were $\Delta V_{a} / \Delta V_{b}=1.51$ and $\Delta I / \Delta V_{b}=-0.21$. This current to piezovoltage modulation ratio thus perfectly matches the value of -0.21 predicted using Eq. (14), based on the $410 \mathrm{~nm}$ ECDL parameters 


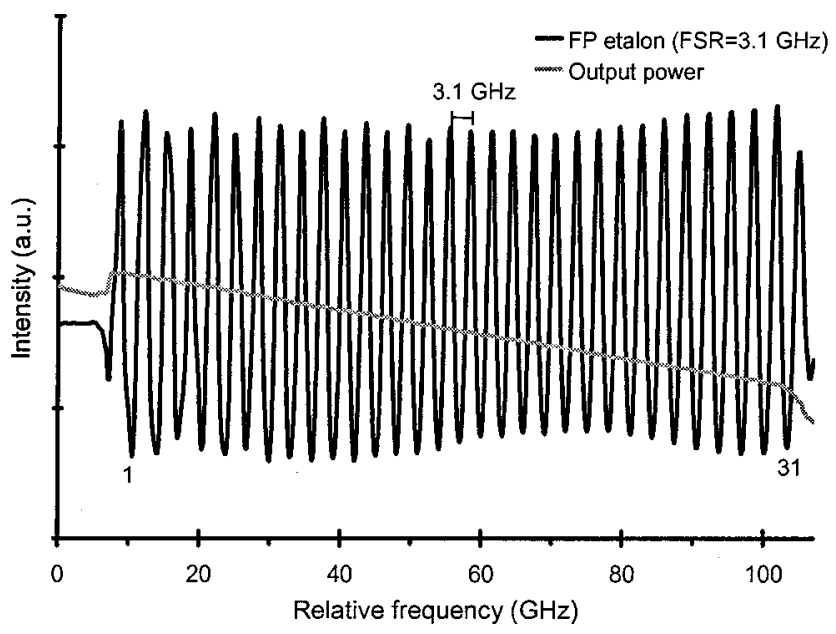

Fig. 8. Transmitted fringe pattern of a $410 \mathrm{~nm}$ extended-cavity diode-laser wavelength scan through a low-finesse Fabry-Perot interferometer (FSR $=3.1 \mathrm{GHz}$ ). 31 fringes can be distinguished, corresponding to a $93 \mathrm{GHz}$ wide mode-hop-free scan. The corresponding laser output power is also shown.

$\left(l_{a}=45.0 \mathrm{~mm}, l_{b}=20.5 \mathrm{~mm}, l_{2}=25.0 \mathrm{~mm}, \alpha=\right.$ $41 \mathrm{~nm} / \mathrm{V}$, and $\beta=4.0 \mathrm{pm} / \mathrm{mA}$ ).

One of the advantages of the multiple-actuator grating configuration, compared to the normal pivotpoint configuration, is the simplicity of independently changing cavity length and grating angle.

At the beginning of an experiment, when adjusting the modulation signal ratios to achieve a mode-hopfree scan, the DC level of the driving voltage for the piezoactuators can be adjusted to optimize the overlap between the extended-cavity mode structure and the FP diode mode. The optimal overlap position is easily identified in practice, as it corresponds to the longest mode-hop-free tuning range. In a similar fashion, either the diode temperature, current, or the grating angle can be used to optimize the initial overlap between the grating-feedback profile and the FP diode mode, whereas in the present ECDL systems this optimization was found to be less critical. Furthermore, small changes made to the relative slopes of the driving voltages could compensate for initial mismatches between the extended cavity, the diode cavity, and the grating. The effects of small nonlinearities or hysteresis of the actuators can also be compensated for by this procedure. We believe that the combination of these effects is part of the explanation for the comparatively long tuning ranges achieved with the ECDL systems described here.

As an example of an application of the $451 \mathrm{~nm}$ ECDL, an absorption spectrum of $\mathrm{Te}_{2}$ vapor was recorded, which is shown in Fig. 9. A single-scan spectrum ( $>60 \mathrm{GHz}$ wide) is shown, which was recorded in a quartz cell containing ${ }^{130} \mathrm{Te}_{2}$ (Opthos Instruments). The cell was heated to $\sim 600{ }^{\circ} \mathrm{C}$, and the pressure in the cell corresponded to the vapor pressure of $\mathrm{Te}_{2}$ at this cell temperature. The spectrum shown in Fig. 9 corresponds to a $100 \mathrm{~mm}$ absorption path length. The laser-induced fluorescence (LIF) spec-

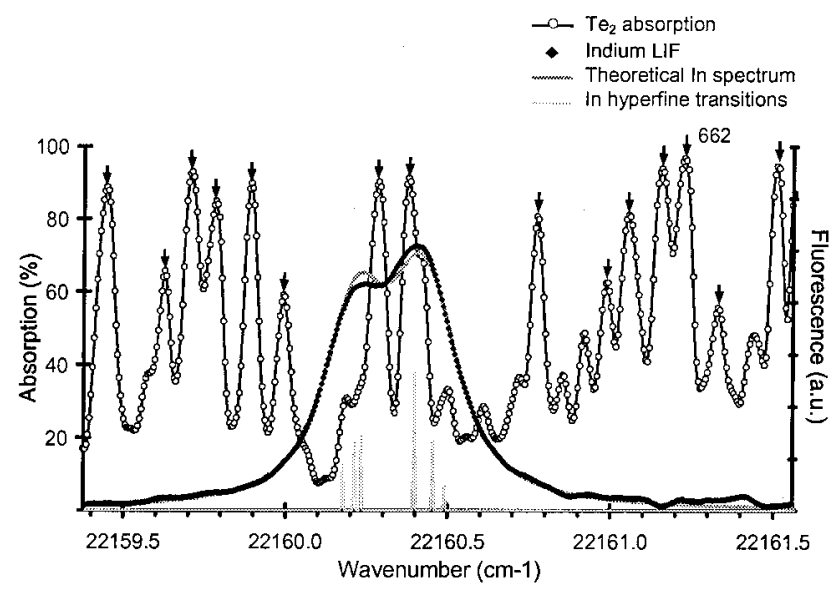

Fig. 9. Simultaneously recorded $\mathrm{Te}_{2}$ absorption and indium LIF spectrum at $\sim 451 \mathrm{~nm}$. The $\mathrm{Te}_{2}$ absorption spectrum was acquired in a low-pressure cell at a temperature of $600{ }^{\circ} \mathrm{C}$. The arrows indicate peaks that could be identified in the tellurium reference atlas. ${ }^{27}$ The LIF spectrum corresponds to the $5^{2} P_{3 / 2} \rightarrow 6^{2} S_{1 / 2}$ transition in indium and was recorded in a flame seeded with indium. A composite fit consisting of six Voigt profiles is shown superimposed on the spectrum. Below the spectrum the positions and relative strengths of the six individual hyperfine transitions are indicated.

trum of the $5^{2} P_{3 / 2} \rightarrow 6^{2} S_{1 / 2}$ transition in neutral indium at $22160.36 \mathrm{~cm}^{-1}$, which is also shown, was recorded simultaneously to provide a reference for the spectral position of the scan. The peak at $22161.22 \mathrm{~cm}^{-1}$ in the $\mathrm{Te}_{2}$ absorption spectrum could thus be identified as line number 662 of the tellurium atlas $\left(\sigma_{662}=22161.2245 \mathrm{~cm}^{-1}\right) .{ }^{27}$ The arrows indicate the peaks in the experimental spectrum that could be identified in the corresponding tellurium atlas spectrum. As seen from this spectrum, molecular tellurium exhibits strong absorption peaks, which would be suitable, for example, in actively locking the wavelength output from ECDL lasers in this spectral region.

The LIF excitation scan spectrum is an average of 200 scans, and was recorded in an atmospheric pressure methane/air Bunsen flame seeded with trace levels of indium atoms. ${ }^{28}$ As the $5^{2} P_{3 / 2}$ and $6^{2} S_{1 / 2}$ states are split into four and two hyperfine levels, respectively, six transitions are observed in total, which appear merged due to pressure and Doppler broadening. ${ }^{29}$ A nonlinear fit of the sum of six Voigt profiles is also shown in Fig. 9. The positions and relative intensities of the six hyperfine transitions are also indicated. The two ECDL systems presented here allow access to both the $5^{2} P_{1 / 2} \rightarrow 6^{2} S_{1 / 2}$ and the $5^{2} P_{3 / 2} \rightarrow 6^{2} S_{1 / 2}$ transitions in indium, at 410 and $451 \mathrm{~nm}$, respectively. ${ }^{29,30}$ This makes diode-laserbased flame-temperature sensing, employing a highresolution two-line atomic fluorescence ${ }^{31}$ (TLAF) technique, possible for the first time. We have recently demonstrated accurate TLAF flame-temperature measurements, using these novel ECDL sources for excitation of indium atoms seeded at trace concentrations to flames. ${ }^{28}$ 


\section{Conclusions}

The combined use of synchronized multiple-actuator grating tuning and injection-current modulation has been described, and has proved capable of achieving long mode-hop-free tuning ranges for single-mode ECDL systems, without the need for using expensive custom-made antireflection-coated laser diodes. Two ECDL systems operating in the blue-violet spectral range were constructed based on this principle using off-the-shelf GaN FP diode lasers. Two systems were developed with center wavelengths of 450 and $410 \mathrm{~nm}$, achieving single-mode tuning ranges exceeding $110 \mathrm{GHz}$ and $90 \mathrm{GHz}$, respectively. These tuning ranges are larger than previously reported ECDL systems employing nonantireflection-coated GaN laser diodes, and even match and exceed those reported using antireflection-coated GaN diodes. These comparatively simple and inexpensive ECDL systems show large promise for applications requiring long single-mode wavelength scans in the blue and violet spectral region.

The optimal relationship between modulation signals applied to the multiple piezoactuators and the diode injection current, to achieve mode-hop-free tuning, has been derived, using a simple geometric model of the extended cavity. The predictions of this model for the ratios between extended-cavity length and diode-current modulations agreed well with the experimentally observed values.

The use of a multiple-actuator design is advantageous, as the normal mechanical optimization of pivot-point location for the grating tuning is replaced by a simple electronic optimization. This makes the ECDL system described here very flexible, as the diode can be replaced by one operating at a different wavelength, or the cavity length can be changed, without any need to modify the system. In this case the only readjustment needed is a new optimization of the ratios of the modulation signals for the actuators and the injection current. Furthermore, the multiple-actuator design also allows for easy optimization of the initial overlap between the gratingfeedback center wavelength and the extended-cavity modes.

This work was supported by a grant from the Royal Society. J. Hult has been supported by a Marie Curie Fellowship of the European Community Human Potential programme, under the contract number HPMF-CT-2002-01574. I. S. Burns has been supported by an EPSRC CASE award, partially funded by Rolls-Royce plc. We also want to thank both P. Ewart and Rolls-Royce plc. for the loan of experimental equipment.

\section{References}

1. C. E. Wieman and L. Hollberg, "Using diode lasers for atomic physics," Rev. Sci. Instrum. 62, 1-20 (1991).

2. D. Sesko, C. G. Fan, and C. E. Wieman, "Production of a cold atomic vapor using diode-laser cooling," J. Opt. Soc. Am. B 5, 1225-1227 (1988).

3. P. W. Werle, P. Mazzinghi, F. D’Amato, M. De Rosa, K. Maurer, and F. Slemr, "Signal processing and calibration proce- dures for in situ diode-laser absorption spectroscopy," Spectrochim. Acta A 60, 1685-1705 (2004).

4. E. Schlosser, J. Wolfrum, L. Hildebrandt, H. Seifert, B. Oser, and V. Ebert, "Diode laser based in-situ detection of alkali atoms: development of a new method for determination of residence-time distribution in combustion plants," Appl. Phys. B 75, 237-247 (2002).

5. M. G. Allen, "Diode laser absorption sensors for gas-dynamic and combustion flows," Meas. Sci. Technol. 9, 545-562 (1998).

6. S. Nakamura, "The roles of structural imperfections in InGaNbased blue light-emitting diodes and laser diodes," Science 281, 956-961 (1998).

7. S. Nagahama, T. Yanamoto, M. Sano, and T. Mukai, "Characteristics of laser diodes composed of GaN-based semiconductor," Phys. Stat. Sol. A 190, 235-246 (2002).

8. K. B. MacAdam, A. Steinbach, and C. E. Wieman, "A narrowband tunable diode laser system with grating feedback and a saturated absorption spectrometer for Cs and Rb," Am. J. Phys. 60, 1098-1111 (1992).

9. L. Ricci, M. Weidemüller, T. Esslinger, A. Hemmerich, C. Zimmermann, V. Vuletic, W. König, and T. W. Hänsch, "A compact grating-stabilized diode-laser system for atomic physics," Opt. Commun. 117, 541-549 (1995).

10. G.-Y. Yan and A. L. Schawlow, "Measurement of diode laser characteristics affecting tunability with an external grating," J. Opt. Soc. Am. B 9, 2122-2127 (1992).

11. F. Favre, D. Le Guen, J. C. Simon, and B. Landousies, "External-cavity semiconductor laser with $15 \mathrm{~nm}$ continuous tuning range," Electron. Lett. 21, 795-796 (1986).

12. M. de Labachelerie and G. Passedat, "Mode-hop suppression of littrow grating-tuned lasers," Appl. Opt. 32, 269-274 (1993).

13. J. Mellis, S. A. Al-Chalabi, K. H. Cameron, R. Wyatt, J. C. Regnault, W. J. Devlin, and M. C. Brain, "Miniature packaged external-cavity semiconductor-laser with $50-\mathrm{GHz}$ continuous electrical tuning range," Electron. Lett. 24, 988-989 (1988).

14. T. Laurila, T. Joutsenoja, R. Hernberg, and M. Kuittinen, "Tunable external-cavity diode laser at $650 \mathrm{~nm}$ based on a transmission diffraction grating," Appl. Opt. 41, 5632-5637 (2002).

15. M. G. Boshier, D. Berkeland, E. A. Hinds, and V. Sandoghdar, "External-cavity frequency stabilization of visible and infrared semiconductor lasers for high resolution spectroscopy," Opt. Commun. 85, 355-359 (1991).

16. L. Hildebrandt, R. Knispel, S. Stry, J. R. Sacher, and F. Schael, "Antireflection-coated blue GaN laser diodes in an external cavity and Doppler-free indium absorption spectroscopy," Appl. Opt. 42, 2110-2118 (2003).

17. C. Petridis, I. D. Lindsay, D. J. M. Stothard, and M. Ebrahimzadeh, "Mode-hop-free tuning over $80 \mathrm{GHz}$ of an extended cavity diode laser without antireflection coating," Rev. Sci. Instrum. 72, 3811-3815 (2001).

18. R. S. Conroy, J. J. Hewett, G. P. T. Lancaster, W. Sibbett, J. W. Allen, and K. Dholakia, "Characterisation of an extended cavity violet diode laser," Opt. Commun. 175, 185-188 (2000).

19. H. Leinen, D. Gläßner, H. Metcalf, R. Wynands, D. Haubrich, and D. Meschede, "GaN blue diode lasers: a spectroscopist's view,” Appl. Phys B 70, 567-571 (2000).

20. U. Gustafsson, J. Alnis, and S. Svanberg, "Atomic spectroscopy with violet laser diodes,” Am. J. Phys. 68, 660-664 (2000).

21. W. R. Trutna and L. F. Stokes, "Continuously tuned externalcavity semiconductor-laser," J. Lightwave Technol. 11, 12791286 (1993).

22. L. Hildebrandt, Sacher Lasertechnik Group, Hannah Arendt Strasse 3-7, D-35037 Marburg, Germany (personal communication, 2004).

23. C. J. Hawthorn, K. P. Webber, and R. E. Scholten, "Littrow configuration tunable external cavity diode laser with fixed 
direction output beam," Rev. Sci. Instrum. 72, 4477-4479 (2001).

24. U. Tisch, B. Meyler, O. Katz, E. Finkman, and J. Salzman, "Dependence of the refractive index of $\mathrm{Al}_{x} \mathrm{Ga}_{1-x} \mathrm{~N}$ on temperature and composition at elevated temperatures," J. Appl. Phys. 89, 2676-2685 (2001).

25. H. X. Jiang and J. Y. Lin, "Mode spacing "anomaly" in InGaN blue lasers," Appl. Phys. Letters 74, 1066-1068 (1999).

26. B. Boggs, C. Greiner, T. Wang, H. Lin, and T. W. Mossberg, "Simple high-coherence rapidly tunable external-cavity diode laser," Opt. Lett. 23, 1906-1908 (1998).

27. J. Cariou and P. Luc, Atlas du Spectre d'Absorption de la Molecule Tellure (Laboratoire Aimé-Cotton, CNRS II, Orsay, France, 1980).
28. J. Hult, I. S. Burns, and C. F. Kaminski, "Two-line atomic fluorescence thermometry using diode lasers," Proc. Combust. Inst. 30, 1535-1543 (2005).

29. I. S. Burns, J. Hult, and C. F. Kaminski, "Spectroscopic use of a novel blue diode laser in a wavelength region around 450 nm," Appl. Phys. B. 79, 491-495 (2004).

30. J. Hult, I. S. Burns, and C. F. Kaminski, "Measurements of the indium hyperfine structure in an atmospheric-pressure flame by use of diode-laser-induced fluorescence," Opt. Lett. 29, 827829 (2004)

31. J. E. Dec and J. O. Keller, "High speed thermometry using two-line atomic fluorescence," Proc. Combust. Inst. 21, 17371745 (1986). 